\title{
Evolution from heterozygous to homozygous KIT mutation in gastrointestinal stromal tumor correlates with the mechanism of mitotic nondisjunction and significant tumor progression
}

Lei L Chen ${ }^{1}$, Joseph A Holden ${ }^{2}$, Haesun $\mathrm{Choi}^{3}$, Jing Zhu ${ }^{4}$, Elsie F Wu ${ }^{4}$, Kimberly A Jones ${ }^{1}$, John H Ward ${ }^{1}$, Robert H Andtbacka ${ }^{5}$, R Lor Randall ${ }^{5}$, Courtney L Scaife ${ }^{5}$, Kelly K Hunt ${ }^{6}$, Victor G Prieto ${ }^{7}$, Austin K Raymond ${ }^{7}$, Wei Zhang ${ }^{7,9}$, Jonathan C Trent ${ }^{8}$, Robert S Benjamin ${ }^{8}$ and Marsha L Frazier ${ }^{4,9}$

${ }^{1}$ Department of Internal Medicine, Division of Hematology/Oncology, Huntsman Cancer Institute, University of Utah, Salt Lake City, UT, USA; ${ }^{2}$ Department of Pathology, Huntsman Cancer Institute, University of Utah, Salt Lake City, UT, USA; ${ }^{3}$ Department of Radiology, The University of Texas MD Anderson Cancer Center, Houston, TX, USA; ${ }^{4}$ Department of Epidemiology, The University of Texas MD Anderson Cancer Center, Houston, TX, USA; ${ }^{5}$ Department of Surgery, Huntsman Cancer Institute, University of Utah, Salt Lake City, UT, USA; ${ }^{6}$ Department of Surgery, The University of Texas MD Anderson Cancer Center, Houston, TX, USA; ${ }^{7}$ Department of Pathology, The University of Texas MD Anderson Cancer Center, Houston, TX, USA; ${ }^{8}$ Department of Sarcoma, The University of Texas MD Anderson Cancer Center, Houston, TX, USA and ${ }^{9}$ The University of Texas Graduate School of Biomedical Sciences at Houston, Houston, TX, USA

Activating mutation in KIT or platelet-derived growth factor- $\alpha$ can lead to gastrointestinal stromal tumors (GISTs). Eighty-four cases from two institutes were analyzed. Of them, $62(74 \%)$ harbored KIT mutations, 7 of which are previously unreported. One exhibited duplication from both intron 11 and exon 11, which has not been reported in KIT in human cancer. A homozygous/hemizygous KIT-activating mutation was found in 9 of the 62 cases $(15 \%)$. We identified three GIST patients with heterozygous KIT-activating mutations at initial presentation, who later recurred with highly aggressive clinical courses. Molecular analysis at recurrence showed total dominance of homozygous (diploid) KIT-activating mutation within a short period of 6-13 months, suggesting an important role of oncogene homozygosity in tumor progression. Topoisomerase II is active in the S- and $G_{2}$ phases of cell cycle and is a direct and accurate proliferative indicator. Cellular and molecular analysis of serial tumor specimens obtained from consecutive surgeries or biopsy within the same patient revealed that these clones that acquired the homozygous KIT mutation exhibited an increased mitotic count and a striking fourfold increase in topoisomerase II proliferative index (percentage cells show positive topoisomerase II nuclear staining compared to the heterozygous counterpart within the same patient. KIT forms a homodimer as the initial step in signal transduction and this may account for the quadruple increase in proliferation. Using SNPs for allelotyping on the serial tumor specimens, we demonstrate that the mechanism of the second hit resulting in homozygous KIT-activating mutation and loss of heterozygosity is achieved by mitotic nondisjunction, contrary to the commonly reported mechanism of mitotic recombination. Modern Pathology (2008) 21, 826-836; doi:10.1038/modpathol.2008.46; published online 16 May 2008

Keywords: homozygous KIT mutation; GIST; SNP

Correspondence: Dr LL Chen, MD, PhD, Department of Internal Medicine, Division of Hematology/Oncology, Huntsman Cancer Institute, University of Utah, 2000 Circle of Hope, Suite 2100, Salt Lake City, UT 84112, USA.

E-mail: lei.chen@hci.utah.edu

Received 15 October 2007; revised 04 February 2008; accepted 10 February 2008; published online 16 May 2008
Gastrointestinal stromal tumors (GISTs) originate from the interstitial cells of Cajal and are driven by a proliferative signal resulting from dominant activating mutations of KIT or platelet-derived growth factor receptor- $\alpha$ (PDGFRA). ${ }^{1-8}$ KIT is important in proliferation, development and maintenance of 
erythropoiesis, differentiation and development of mast cells, melanogenesis, spermatogenesis, gastrointestinal (GI) pacemaker activity, and coordination with myenteric neurons and smooth muscle cells in GI peristalsis.

Imatinib (STI571, Gleevec, Glivec, Novartis Pharmaceuticals $)^{9}$ is a small molecule-selective inhibitor of the tyrosine kinases ABL, BCR-ABL, KIT, PDGFRA, and PDGFRB. It is the first effective targeted cancer therapy and has revolutionized the treatment of chronic myelogenous leukemia and GIST. ${ }^{10,11}$ Resistance to imatinib has emerged, most commonly because of a new second (or acquired) mutation in the kinase domain. ${ }^{12-16}$

GISTs show a predilection for liver metastasis and peritoneal implants-in vivo clones, and therefore provide an excellent model for studying the tumor biology. The histologic types of GISTs vary from very low grade to highly aggressive, and mitotic count vary more than 20-fold, To understand tumor progression, longitudinal follow-up of clonal evolution and comparison of primary tumor and subsequent recurrences within the same patient is probably the best means of circumventing both the interpatient variations and the complex events associated with late-stage advanced tumor progression.

Multiple genetic events are involved in initiation and progression of human cancer. While two hits to achieve loss of heterozygosity ( $\mathrm{LOH}$ ) are necessary to inactivate a tumor suppressor gene, ${ }^{17,18}$ one hit of an activating oncogene mutation is considered to be sufficient for the initiation of neoplasia. $\mathrm{LOH}$ has been increasingly observed in many human neoplasms, ${ }^{19-22}$ but its significance and mechanism of action in many common advanced tumors remain unclear. The frequency of LOH resulting in acquisition of a homozygous or a hemizygous KIT oncogene mutation in GISTs has been reported ranging from 4 to $18 \%$ with lower frequency noted in populationbased study and higher frequency in cohorts in major cancer center. ${ }^{3,8,23-25}$ The GIST clones with LOH acquiring homozygous KIT mutation prevail and dominate, strongly suggesting that they provide proliferative and metastatic advantages. However, little is known about the significance and mechanism of $\mathrm{LOH}$ acquiring homozygosity of an oncogene in tumorigenesis.

Examination of single nucleotide polymorphisms (SNPs) - the most abundant class of human polymorphisms-has proven highly valuable in forensic medicine. SNPs also are under study as a tool for revealing genetic variations that could influence health and disease, ${ }^{26}$ notably the International HapMap Project (http://www.hapmap.org/). SNPs are archived in the National Center for Biotechnology Information SNP database and the National Cancer Institute Cancer Genome Anatomy Project SNP500 cancer databases, among others. SNPs and variants, which represent the SNPs that have not been reported or archived yet, are valuable tools for allelotyping to study clonal evolution in vivo during the progression of GISTs.

Here we present the data of our two-institute study of 84 patients with GISTs. We discovered seven new KIT mutations, including an unusual mechanism of such mutation. With longitudinal follow-up within the same patient, we show that evolution from heterozygous to homozygous KIT oncogene mutation results in total dominance of the latter, occurs in $4-18 \%$ of GISTs, ${ }^{3,8,23-25}$ manifests increased mitotic count, a dramatic fourfold increase in topoisomerase II proliferative index, and clinically correlates with significant tumor progression. Furthermore, our study using SNPs showed that nondisjunction, rather than mitotic recombination, is the mechanism to achieve the homozygous status of KIT mutation.

\section{Materials and methods}

\section{Tumor Specimens}

All GISTs (surgical specimens, biopsies, paraffinembedded tissue) were obtained for study with the approval of the investigational review boards of Huntsman Cancer Institute, University of Utah, Salt Lake City, UT, USA (protocol IRB00022044), and The University of Texas MD Anderson Cancer Center, Houston, TX, USA (laboratory protocol LAB02-433). Specimens were processed in a routine manner for histologic and immunohistochemical examination at the time of diagnosis. Mitotic counts were performed as number of mitotic figures per 50 high-powered fields (HPF).

\section{DNA and cDNA Sequence Analysis}

DNA was isolated from frozen tissue by using a QIAamp DNA mini kit (Qiagen Inc., Valencia, CA, USA) according to the manufacturer's instructions; it was also isolated from paraffin-embedded tissue. After we reviewed the H\&E-stained slides, we identified the areas of tumor on the unstained slides and microdissected them off the slides. The DNA was isolated by proteinase $\mathrm{K}$ digestion and then heating in boiling water to inactivate the proteinase $\mathrm{K}$, and the lysate was used for polymerase chain reaction (PCR) analysis. RNA was extracted from the frozen tissue using standard methods and an RNeasy minicolumn according to the manufacturer's instructions (Qiagen). The complementary DNA (cDNA) was prepared by using Two-step TaqMan Reverse Transcription Reagent (Applied Biosystems, Foster City, CA, USA) according to the manufacturer's instructions, except that instead of using random primers, a primer specific for KIT RNA was used (KIT 2961R, 5'-TTCCTGGAGGGGTGACC CAAACACT). The CDNA was then subjected to PCR analysis. Nucleotide sequencing was performed using a 3730X1 DNA Analyzer from Applied 
Biosystems at the MD Anderson Cancer Center Nucleic Acid Core Facility, and at the ARUP Institute for Clinical and Experimental Pathology, University of Utah. The primer sequences for KIT genomic and cDNA-sequencing analysis were performed as described before. ${ }^{12,27}$

\section{IHC Analysis on Paraffin Sections}

Antibody against human DNA topoisomerase II- $\alpha$ was developed at the University of $\mathrm{Utah}^{27}$ and was used at a dilution of 1:1000. Topoisomerase II index is the percentage of cells that show a positive nuclear stain. At least 500 GIST cells were counted for this analysis, and all topoisomerase II index indices were determined by 1 investigator (JAH).

\section{Single Nucleotide Polymorphisms}

The primer sequences for the SNPs and variants are listed in Table 1.

\section{Results}

\section{Mutational Analysis of KIT and PDGFRA in 84 GISTs}

We performed sequencing analysis of KIT and PDGFRA on 84 GISTs from 1998 to 2007, 69 from MD Anderson Cancer Center and 15 from Huntsman Cancer Institute. This report concentrates on our studies related to KIT mutation. KIT mutations were detected in 62 of the 84 (74\%) GISTs. Among the 62 GISTs harboring KIT mutations, KIT exon 11 mutations were detected in 51 of $62(82 \%)$, KIT exon 9 A503-Y504dup was found in 10 of $62(16 \%)$, and exon 17 mutation was found in 1 of 62 (1.6\%) GISTs. After a close examination of the 62 GISTs harboring KIT mutations, 3 were proven to be homozygous, and 6 were either hemizygous or homozygous mutations; together, they constituted $15 \%$ of all GISTs harboring KIT mutations. We identified 7 KIT exon 11 mutations (Table 2) that were new to the
Sanger Institute Catalogues of Somatic Mutation in Cancer database (http://www.sanger.ac.uk/perl/ genetics/CGP/cosmic?action $=$ bygene\&ln $=$ KIT\&start $=1 \&$ end $=977 \&$ coords $=\mathrm{AA}: \mathrm{AA}$ ).

Table 2 shows seven new mutations of KIT in GISTs. We identified an unusual mechanism of mutation that has never been reported in KIT in any human cancer before (Table 2, no. 7). Insertion of the first two nucleotides of intron 11 (gt) plus duplication of nucleotides 1750-1795 in exon 11 led to the insertion of a ' $G$ ' plus duplication of PYDHKWEFPRNRLSF between codons 591 and 592 as illustrated in Figure 1. The sequencing analysis of cDNA showed the same mutation.

Evolution from Heterozygous to Homozygous (Diploid) KIT-Activating Mutation Results in Increased Mitotic Counts, Fourfold Increase in Topoisomerase II Proliferative Index, and Significant Tumor Progression

Through longitudinal clinical follow-up and molecular studies on our serial surgical and biopsy specimens, we identified three GIST patients whose first surgical specimens showed a heterozygous KITactivating mutation but later progressed and the subsequent second surgical specimens demonstrated total dominance of what could initially be interpreted as either a 'homozygous' or 'hemizygous' KIT-activating mutation within a short duration of 8-13 months, suggesting a highly selective advantage of those clones over their heterozygous counterparts. When imatinib became available, all three patients were treated with it and had an excellent initial response, but later they became imatinib resistant and underwent either subsequent biopsy or surgery.

Patient A presented with a primary gastric GIST without nodal or distant metastasis and underwent surgery with curative intent. Sequencing analysis revealed a heterozygous KIT exon 11 deletion of $6 \mathrm{bp:}$ (GGAAGG1691-1696del (W557_V559>F)) (Figure 2a-2). The GIST recurred 13 months later

Table 1 Single nucleotide polymorphisms used as allelic markers

\begin{tabular}{|c|c|c|c|}
\hline & SNP or variant & Primer sequence & Gene/chromosome mapping \\
\hline 1 & SNP-rs4253320 & $\begin{array}{l}\text { F-5'-CTTGAGGCCAGGAGTTTGAG } \\
\text { R-5'-CTTGCCCGAGGTCACATAAT }\end{array}$ & KLKB1 (chromosome $4 \mathrm{q}^{34-35}$ ) \\
\hline 2 & SNP-rs17464616 & $\begin{array}{l}\text { F-5'-GGCTCTGATCTGCCAAACTC } \\
\text { R-5'-AGGCACACAATATGGCAAAG }\end{array}$ & STK32B (chromosome $4 \mathrm{p}^{16.2}$ ) \\
\hline 3 & SNP-rs362273 & $\begin{array}{l}\text { F-5'-GAGCTTTTCTCCTGGGTGTG } \\
\text { R-5'-AGACTCAGGGTGCTGGTGTT }\end{array}$ & Huntington gene (chromosome $4 \mathrm{p}^{16.3}$ ) \\
\hline 4 & Variant in intron 16 of $K I T$ & $\begin{array}{l}\text { F-5'-GTGAACATCATTCAAGGCGT } \\
\text { R-5'-CCTTTGCAGGACTGTCAAGCA }\end{array}$ & $\begin{array}{l}\text { KIT (chromosome } 4 \mathrm{q}^{12} \text { ), } 76 \text { bp } 5^{\prime} \\
\text { from KIT exon } 17\end{array}$ \\
\hline 5 & Variant in intron 1 of $K I T$ & $\begin{array}{l}\text { F (at nucleotide position 2866660), } \\
\text { F-5'-AGTTGGAAACTGTGTGCCAG } \\
\text { R (at nucleotide position 2866943), } \\
\text { R-5'-GTGACATTGGCCATCCTGT }\end{array}$ & $\begin{array}{l}\text { KIT (chromosome } 4 \mathrm{q}^{12} \text { ), chromosome } \\
4 \text { contiguity } \\
\text { NT_022853 [gi:29789926] nucleotide } \\
\text { position } 2866845\end{array}$ \\
\hline
\end{tabular}

SNP, single nucleotide polymorphism. 
Table 2 Seven new KIT mutations detected in GISTs

\begin{tabular}{|c|c|c|c|}
\hline & KIT mutation & KIT mutation & Amino acids deduced from mutation \\
\hline & Wild-type $K I T$ & Wild-type $K I T$ & 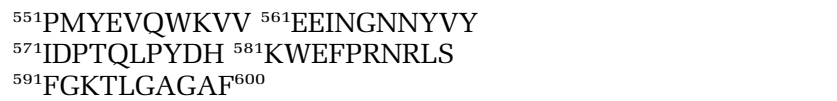 \\
\hline 1 & $1763 \mathrm{~A}>\mathrm{G}$ & K581R & $\begin{array}{l}{ }^{561} \text { EEINGNNYVY }{ }^{571} \mathrm{IDPTQLPYDH}^{580} \\
{ }^{581} \text { RWEFPRNRLS }\end{array}$ \\
\hline 2 & 1695_1730del & K558_Y570>N & $\begin{array}{l}{ }^{551} \text { PMYEVQW--- }{ }^{561}---------N \\
{ }^{571}{ }^{5 D P T Q L P Y D H}{ }^{580}\end{array}$ \\
\hline 3 & $1692 \mathrm{G}>\mathrm{C}$, and $1694 \mathrm{~A}>\mathrm{C}$ & W557_K558>CT & 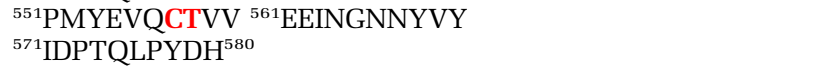 \\
\hline 4 & 1688del, and 1690_1694del & Q556_K558>R & $\begin{array}{l}{ }^{551} \mathrm{PMYEV}--\mathrm{RVV}{ }^{561} \mathrm{EEINGNNYVY} \\
{ }^{571} \mathrm{IDPTQLPYDH}^{580}\end{array}$ \\
\hline 5 & $\begin{array}{l}\text { 1687_1737 } \\
\text { delinsCCATCC }\end{array}$ & Q556_D572>PS & $\begin{array}{l}{ }^{551} \text { PMYEV---_-- }{ }^{561}---------- \\
{ }^{571} \text { PSPTQLPYDH }\end{array}$ \\
\hline $\begin{array}{l}6 \\
7\end{array}$ & $\begin{array}{l}\text { 1753_1761dup } \\
\text { GT1795_1796ins }\end{array}$ & YDH580-581dup & ${ }^{571} \mathrm{IDPTQLP}$ YDH $\mid \mathrm{YDH}{ }^{581} \mathrm{KWEFPRNRLS}{ }^{590}$ \\
\hline & $\begin{array}{l}\text { dup } 1750-1795 \\
\text { (refer to Figure 1) }\end{array}$ & $\begin{array}{l}\text { GPYDHKWEFPRNRLSF } \\
\text { 591-592insdup }\end{array}$ & $\begin{array}{l}{ }^{571} \mathrm{IDPTQL} \\
{ }^{592} \mathrm{GKTLH}{ }^{581} \mathrm{KWEFPRNRLS}{ }^{591} \mathrm{~F}\end{array}$ PYDHKWEFPRNRLSF \\
\hline
\end{tabular}

Red color letter: mutations; ---: deletion; red color in red box: duplication; black color in black box: wild-type sequences that are duplicated.

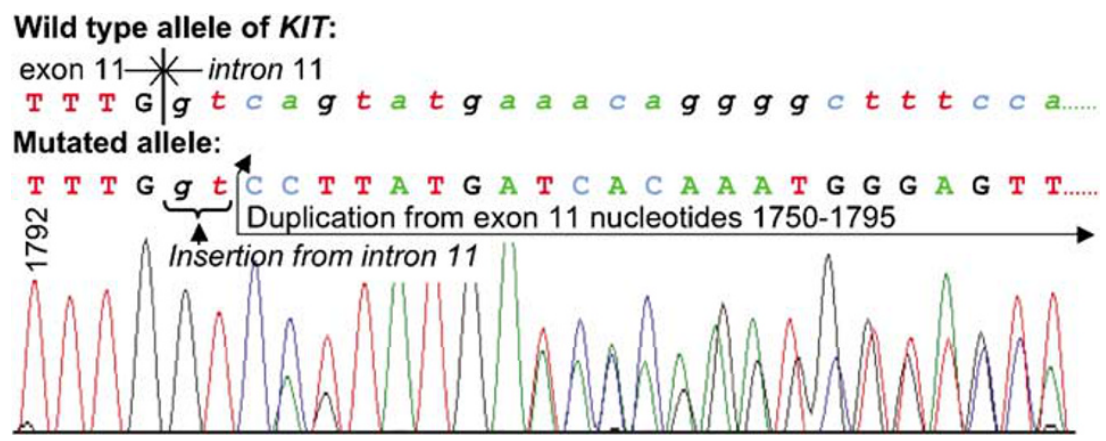

Figure 1 An unusual KIT mutation in a patient with GIST exhibiting GPYDHKWEFPRNRLSF591-592insdup. The chromatogram reveals an unusual mechanism that has never been reported in human KIT before. It involves insertion of 2 nucleotides from intron 11 plus internal duplication of 46 nucleotides (1750-1795) from exon 11. Genomic DNA and cDNA-sequencing analysis showed identical results. This is case no. 7 as shown in Table 2.

with a perigastric mass and a liver lesion and were resected with clean margins in the pre-imatinib era. Sequencing analysis revealed total dominance of homozygous or hemizygous $6 \mathrm{bp}$ deletion (GGAAGG1691-1696del) (Figure 2a-3). Patient A recurred again with numerous liver lesions and extensive peritoneal implants (Figure 3a) within a short 51/2 months indicating an extremely aggressive clinical course. Patient A was then enrolled in S0033 imatinib clinical trial and had a swift and near-complete response (Figure 3b) lasting for 6 months, followed by imatinib resistance (Figure 3c-e, arrow). The imatinib-resistant implant again showed very rapid progression with an estimated doubling time of 35 days, and the biopsy revealed the same homozygous 6 bp deletion (GGAAGG16911696del) plus a new second 'heterozygous' KIT mutation, 1982T $\rightarrow \mathrm{C}$ (V654A) in exon 13, which conferred imatinib resistance. ${ }^{12}$ The emphasis here is on the heterozygous new second KIT mutation, which proves the presence of two alleles and thus provides unequivocal evidence of the homozygous, not hemizygous, status of the KIT-activating mutation.

This unusual aggressive clinical course of patient A prompted us to compare the histology, mitotic counts, and topoisomerase II activity. Mitosis represents a very small fraction of the cell cycle and may be lost during fixation. Topoisomerases II controls DNA topology by inducing DNA double-strand breaks and is crucial in chromosome condensation and segregation before cell division in proliferating cells. Topoisomerases II is synthesized in late $G_{1}$, present throughout $S$ phase, and then degraded after mitosis, and thus represents a highly reliable measure of proliferation. The same paraffin blocks from the first and second surgeries were used for sequencing analysis, $\mathrm{H} \& \mathrm{E}$, mitotic count, and IHC for topoisomerase II analysis. The chromatogram of the first surgical specimen exhibited heterozygous 

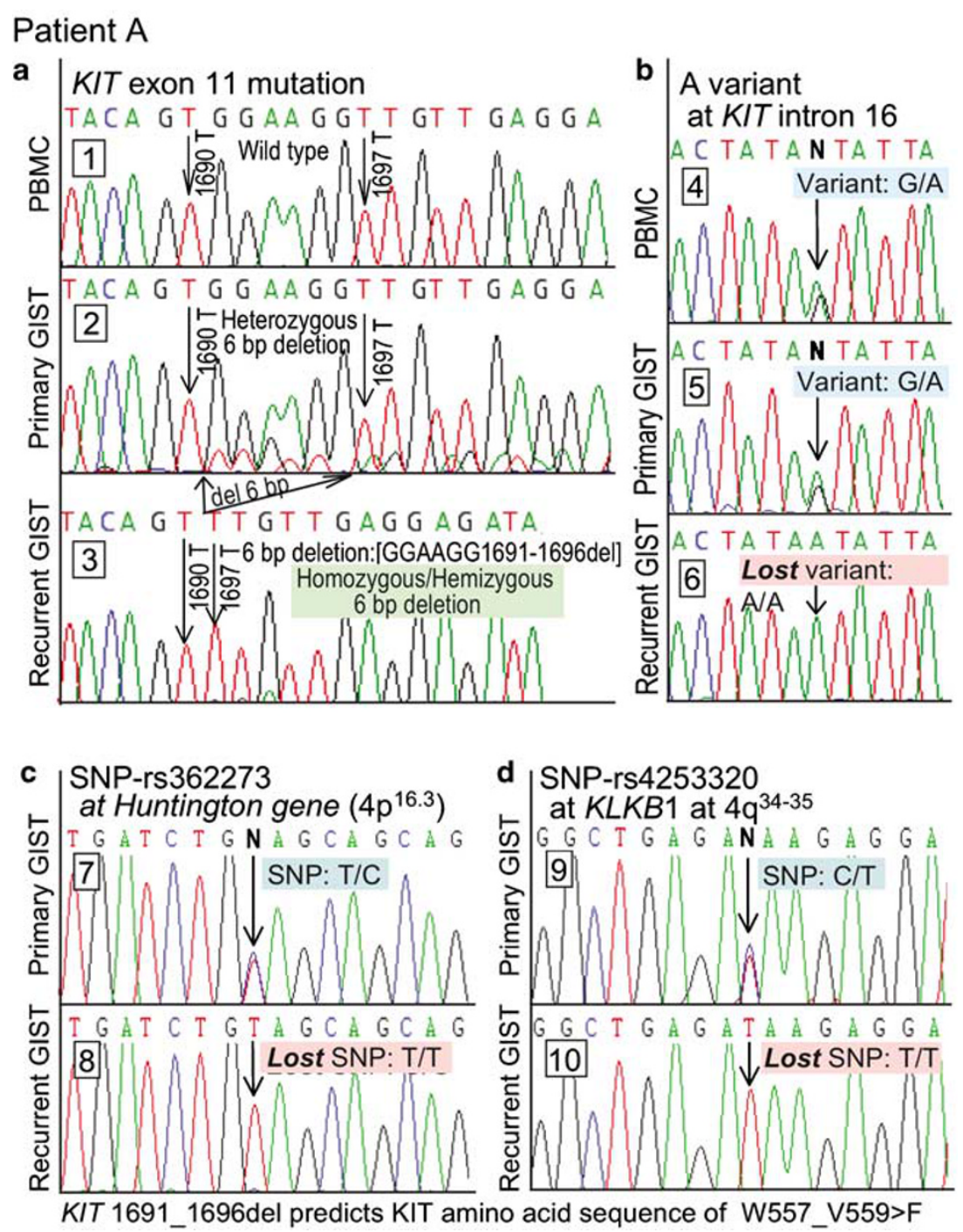

Figure 2 Evolution from a heterozygous to a homozygous KIT-activation mutation of a 6-bp deletion in exon 11 and loss of SNPs in the recurrent GISTs of patient A. (a-1) PBMCs show the wild-type sequence. (a-2) Primary GIST shows a heterozygous 6-bp deletion: (GGAAGG1691-1696del) in KIT exon 11. (a-3) Recurrent GIST shows a homozygous or a hemizygous 6-bp deletion: (GGAAGG16911696del). (b-4) PBMCs show a variant in KIT intron 16. (b-5) Primary GIST demonstrates the polymorphism of the variant. (b-6) Recurrent GIST shows loss of the variant. (c-7) Primary GIST shows SNP-rs362273 in the Huntington gene at $4 \mathrm{p}^{16.3}$. (c-8) Recurrent GIST shows loss of SNP-rs362273. (d-9) Primary GIST shows SNP-rs4253320 in KLKB1 at chromosome $4 q^{34-35}$. (d-10) Recurrent GIST shows loss of SNP-rs4253320.

6 bp deletion (Figure 2a-2). The H\&E and IHC for topoisomerase II are shown in Figure $4 \mathrm{a}$ and $\mathrm{b}$, respectively. The mitotic count was 17/50 HPF, and the topoisomerase II proliferative index (fraction of cell showing positive nuclear staining) was 12. The chromatogram of the second surgical specimen exhibits homozygous $6 \mathrm{bp}$ deletion (Figure 2a-3). The H\&E and topoisomerase II IHC are shown in Figure 4c and d, respectively. The mitotic count was 25/50 HPF, and the topoisomerase II proliferative index is 49, a significant fourfold increase compared to the heterozygous counterpart. Comparison of Figure $4 \mathrm{~b}$ and $\mathrm{d}$ reveals striking differences in topoisomerase II nuclear staining and correlates well with the highly aggressive clinical course of the second recurrence and the subsequent extremely rapid growth of the imatinib-resistant clone (Figure 3c-e).

Patient B presented with GIST harboring a heterozygous 6-bp internal tandem duplication in KIT exon 9: (GCCTAT1525-1530dup (A502_Y503dup)) (Figure 5a-2) and underwent surgery. The GIST recurred 8 months later and the recurrent tumors were surgically removed. DNAsequencing analysis showed evolution from the heterozygous to a homozygous 6-bp internal tandem duplication in KIT exon 9 (Figure 5a-3). Histology showed doubling in mitotic count and a striking fourfold increase in topoisomerase II proliferative index (data not shown). 

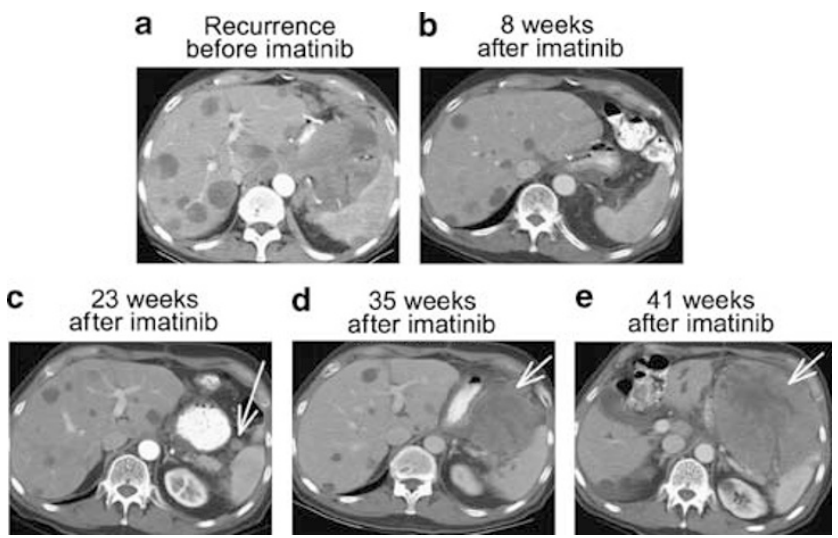

Figure 3 CT images of patient A. (a) Recurrence with liver metastasis and extensive peritoneal implants, (b) swift and near-complete response to imatinib, (c) emergence of an imatinib-resistant clone, (d, e) rapid tumor progression.

Patient $\mathrm{C}$ showed evolution from a heterozygous to homozygous missense $1690 \mathrm{~A} \rightarrow \mathrm{C}(\mathrm{W} 557 \mathrm{G})$ activating-KIT exon 11 mutation along with doubling of mitotic count and fourfold increase in topoisomerase proliferative index (data not shown).

Using GIST model and topoisomerase II proliferative index, we demonstrated that acquisition of a homozygous-activating oncogene mutation with $\mathrm{LOH}$ provides a fourfold increase in proliferative advantage and leads to total dominance and corresponding shift to an aggressive clinical course. Acquisition of a homozygous KIT-activating mutation represents an important mechanism of tumor progression in $4-18 \%$ of GISTs. ${ }^{3,8,23-25}$

\section{Mitotic Nondisjunction Leads to Evolution from a Heterozygous to a Homozygous KIT Mutation with LOH In Vivo in Three GIST Patients}

The possible mechanisms leading to homozygosity of KIT mutation in GISTs include (1) a second hit on the normal allele, resulting in the same KIT-activating mutation, (2) gene conversion through locusrestricted recombinational events, (3) epigenetic allelic silencing, (4) mitotic recombination, a process of reciprocal exchange of genetic material between nonsister chromatids of homologous chromosomes in mitotic cells, yielding partial uniparental disomy of chromosome 4 harboring the mutated KIT, and (5) mitotic nondisjunction (ie, nonsegregation of sister chromatids) causing uniparental isodisomy.

To distinguish among these five mechanisms, we examined many SNPs and variants for allelotyping, including introns of KIT $\left(4 \mathrm{q}^{12}\right.$, in very close proximity to the centromere), the Huntington gene $\left(4 \mathrm{p}^{16.3}\right.$, at the tip of $\left.4 \mathrm{p}\right)$, the STK32B gene $\left(4 \mathrm{p}^{16.2}\right.$, at the tip of $4 p)$, and the $K L K B 1$ gene $\left(4 q^{34-35}\right.$, at the tip of chromosome 4q). We first searched for SNPs and variants on germ-line DNA from peripheral blood mononuclear cells (PBMCs) of these three patients, and then tested the SNPs and variants on the primary GIST (with a heterozygous KIT-activating mutation), the recurrent GIST (with a homozygous KIT-activating mutation), and the imatinib-resistant GIST (with a homozygous KIT-activating mutation). If mechanisms (1), (2), or (3) were correct, both the paternal and maternal chromosomes 4 would exist, and the SNPs and variants that are mapped to the tip of $4 p$ and $4 q$ would be preserved in the recurrent GIST and the imatinib-resistant GIST. Mechanism (1) can be further differentiated from (2) and (3) by the SNPs and variants in introns of KIT because mechanism (1) would preserve all SNPs and variants in KIT introns, whereas mechanisms (2) and (3) would result in the loss of all SNPs and variants in KIT introns. If mechanism (4) were true, we would expect the loss of the SNPs and variants that are mapped to introns of KIT plus the loss of SNPs and variants located in either the $4 p$ or $4 q$ end of the chromosome, but not both, in the recurrent and imatinib-resistant GISTs. If mechanism (5) were true, the entire chromosome 4 harboring the wildtype KIT would be lost, and we would expect the loss of all SNPs and variants in the recurrent and imatinib-resistant GISTs.

The PBMCs and primary GIST of patient A demonstrated polymorphisms of a variant in KIT intron 16 (Figure 2b-4, b-5), SNP-rs362273 (Figure 2c-7) in the Huntington gene at $4 \mathrm{p}^{16.3}$, and SNP-rs4253320 (Figure 2d-9) in the KLKB1 gene at $4 q^{34-35}$. When the first recurrent GIST was tested, we observed the loss of all polymorphisms in KIT intron 16 (Figure 2b-6), SNP-rs362273 (Figure 2c-8) in the Huntington gene at $4 \mathrm{p}^{16.3}$, and SNP-rs4253320 (Figure $2 \mathrm{~d}-10$ ) in the $K L K B 1$ gene at $4 \mathrm{q}^{34-35}$.

The PBMCs and primary GIST of patient B demonstrated a variant in intron 1 of KIT (Figure 5b-4, b-5) , an SNP-rs17464616 (Figure 5c-7) in $S T K 32 B$ at $4 \mathrm{p}^{16.2}$, and an SNP-rs4253320 (Figure 5d-9) in $K L K B 1$ at $4 \mathrm{q}^{34-35}$. All polymorphisms were no longer detectable in the recurrent and imatinib-resistant GISTs (Figure 5b-6, c-8, d-10).

These data obtained from patients $\mathrm{A}$ and $\mathrm{B}$ demonstrate $\mathrm{LOH}$ in both ends of chromosome $4 p$ and $4 \mathrm{q}$ and KIT strongly supporting the mechanism (5) of mitotic nondisjunction, and conclusively ruling out mechanisms (1-4). The data from patient C (not shown here) also supported the mechanism of mitotic nondisjunction as the mechanism in achieving homozygous KIT mutation.

\section{Discussion}

Our study on 84 GIST cases provides valuable insight into the biology of these tumors. Seven new KIT mutations were discovered (Table 2), including case no. 7 that is the first to show an insertion of nucleotides from intron 11 separating the two internal duplications of exon 11 of KIT in 

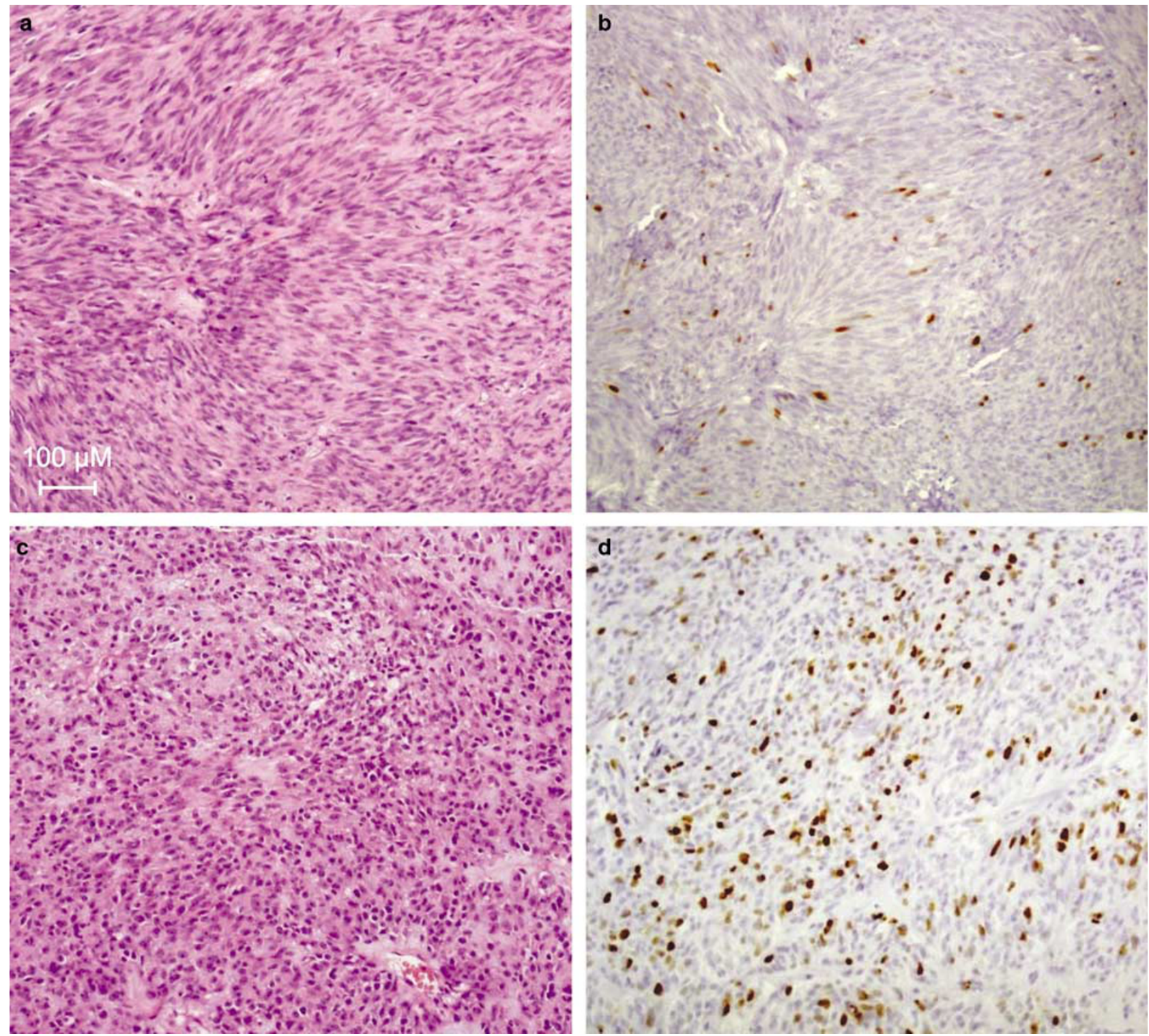

Figure $4 \mathrm{H} \& \mathrm{E}$ and IHC of topoisomerase II $\alpha$. The H\&E of the primary GIST and recurrent GIST of patient A are shown in (a) and (b), respectively. The proliferative index is defined as the percentage of cells exhibiting positive topoisomerase II nuclear staining. Topoisomerase II proliferative index shows a fourfold difference comparing the primary GIST exhibiting heterozygous KIT-activating mutation (c) with the recurrent GIST exhibiting homozygous KIT-activating mutation (d).

GISTs. Internal tandem duplications of KIT exon 11 in GISTs have been reported to represent $4-7 \%$ of KIT mutations and correlate with a favorable clinical course. ${ }^{28}$ internal tandem duplication of exon 12 of KIT was reported in childhood acute myeloid leukemia. ${ }^{29}$ The most common internal tandem duplication of KIT is AY502-502dup of KIT exon 9 in GISTs. Case no. 7 (Table 2) represents the first case of this unusual mechanism of insertion plus duplication involving both KIT exon 11 and intron 11 in GIST. This mechanism, although rare, has been reported in other molecules and other malignancies. For example, there was one reported case of canine spontaneous mast cell tumors that demonstrated an insertion of $9 \mathrm{bp}$ from KIT intron 11 plus internal duplication of exon $11 .^{30}$ In addition, a couple of insertions in FLT3 spanning the exon-intron boundary have been reported in acute myeloid leukemia. ${ }^{31,32}$

We analyzed the GIST implants-the equivalent of in vivo clones-within the same patient at the time of the first surgery (specimens were cryopreserved in tissue bank), at the time of multiple recurrences, and at the time of developing imatinib resistance. Although this unique approach took 5 years to obtain longitudinal follow-up on three cases, it has the tremendous advantage of circumventing both the interpatient variations and the unknown complex events associated with each individual's endstage tumor progression, thus providing conclusive 


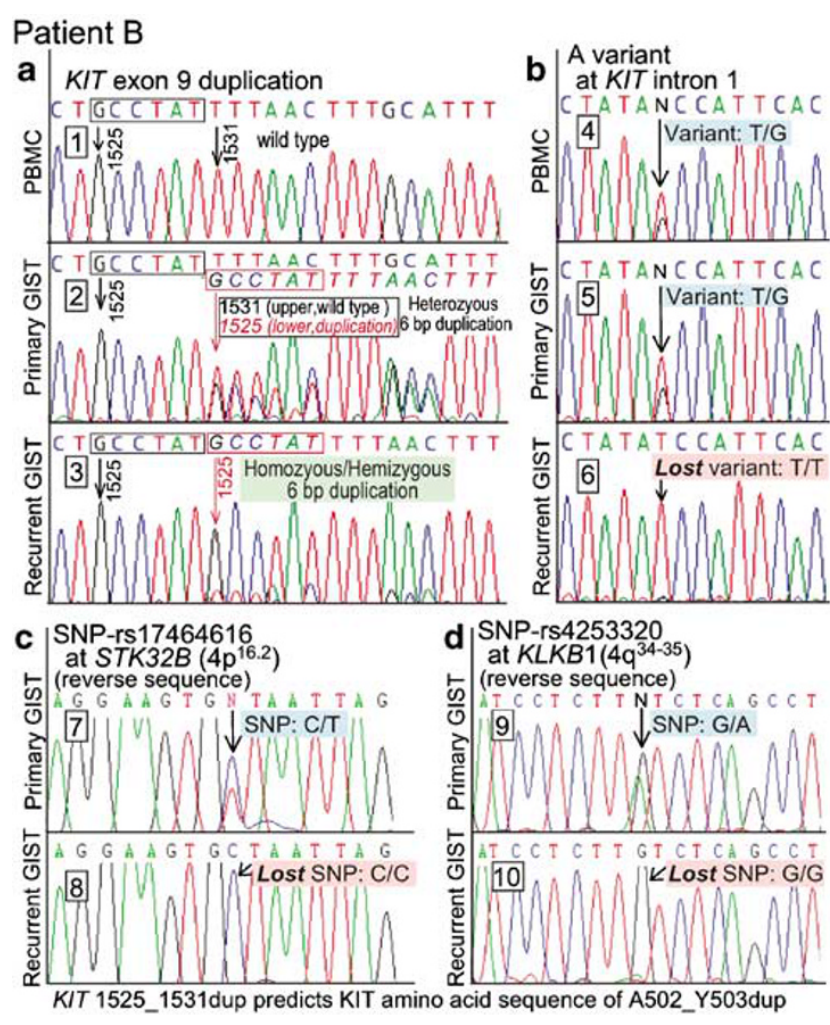

Figure 5 Evolution from a heterozygous to a homozygous KITactivating 6-bp duplication in exon 9 and loss of SNPs in the recurrent GISTs of patient B. (a-1) PBMCs show wild-type sequence. (a-2) Primary GIST shows a heterozygous 6-bp duplication in KIT exon 9: (GCCTAT1525-1530dup). (a-3) Recurrent GIST shows a homozygous or a hemizygous 6-bp duplication in KIT exon 9: (GCCTAT1525-1530dup). (b-4) PBMCs show a variant in KIT intron 1. (b-5) Primary GIST shows the presence of the variant. (b-6) Recurrent GIST shows loss of the variant. (c-7) Primary GIST shows polymorphism of SNP-rs17464616 in STK32B at chromosome $4 \mathrm{p}^{16.2}$. (c-8) Recurrent GIST shows the loss of SNP-rs17464616. (d-9) Primary GIST shows the polymorphism of SNP-rs4253320 in KLKB1 at chromosome $4 \mathrm{q}^{34-35}$. (d-10) Recurrent GIST shows the loss of SNP-rs4253320.

evidences. We identified three GIST patients who experienced rapid progression in short 6-13 months, examination of their tumors revealed evolution from an initial heterozygous KIT mutation to total dominance of a homozygous KIT mutation at the time of recurrence and fourfold increase in topoisomerase proliferative index when comparisons were made with the heterozygous counterpart within the same patient.

Homozygous or hemizygous KIT mutations occurred between 4 and $18 \%$ of GISTs ${ }^{3,8,23-25}$ and were found mainly in metastatic GIST. ${ }^{33}$ The three GIST patients initially presented with heterozygous KIT mutation with 1:1 ratio of the normal and the KIT-activating mutation, and later evolved to homozygous KIT mutation by the mechanism of nondisjunction. They subsequently developed imatinib resistance acquiring a second new mutation and the chromatograms exhibit a 1:1 ratio of normal and a missense mutation that confer imatinib resistance. These data are suggestive of 'diploid' homozygous rather than hemizygous or pseudo-homozygous status, which is a rare condition first described in familial hypercoagulable disease, and is characterized by the co-inheritance of the heterozygous Factor V Leiden mutations and a Factor V null mutation on different alleles. ${ }^{34,35}$ A previous study by Lasota et $a 1^{24}$ examined the nature of homozygous KIT exon 11 mutation using FISH studies in 17 GISTs and reported that most of these 17 cases show homozygous rather than hemizygous status, consistent with our findings. The normal cells of patients with familial GIST exhibit a germ-line heterozygous KIT mutation. However, the large and more aggressive GISTs of familial GIST patients often harbor a homozygous KIT mutation, ${ }^{36,37}$ further supporting our findings. Although these three patients bear large tumor burden, of note, homozygous KIT mutation has been reported in small minimal GISTs. $^{38}$

Upon ligand binding, KIT first forms a homodimer, followed by autophosphorylation of several tyrosine residues of KIT. Homodimers containing mutated KIT in both alleles likely contribute the majority of the constitutive proliferative signal in GISTs. Tyr568 of KIT (pY568KIT) is the initial and primary autophosphorylation site of KIT. ${ }^{39}$ We showed that pY568KIT, but not other phosphorylated tyrosines, localizes in nucleus of GIST.40 Further studies (LL Chen, unpublished data) show that the percent of nuclear localization of pY568KIT increased fourfold in the homozygous GISTs compared to the heterozygous counterpart within the same patients, in parallel with the fourfold increase in topoisomerase II proliferative index. When GIST exhibits a heterozygous KIT mutation, $50 \%$ of the homodimers are paired with one mutated KIT and one wild-type KIT, and only ' $25 \%$ ' of homodimers are paired with both mutated KIT. In contrast, when the GIST evolves to homozygous KIT mutation, ' $100 \%$ ' homodimers are paired with mutated KIT, and can potentially transmit a fourfold increase in KIT signaling compared to the same patient's heterozygous counterpart. This simple model offers a logical explanation of fourfold increase in topoisomerase proliferative index and a change to a much more aggressive phenotype during evolution from heterozygous to homozygous KIT-activating mutation, although additional concomitant unknown etiology or loss of a tumor suppressor gene cannot be ruled out.

$\mathrm{LOH}$ has been increasingly observed in many human neoplasms. Most of the first hits are intragenic mutations, but the mechanisms of the second hits, leading to allelic $\mathrm{LOH}$, are more complex. For example, a mouse model of familial adenomatous polyposis showed homologous somatic recombination was the principal pathway resulting in $\mathrm{LOH}$ of the $A P C$ tumorsuppressor gene. ${ }^{41,42}$ Gene conversion was reported as a frequent mechanism of inactivation of the wild-type allele in cancers from carriers of the 
MLH1/MSH2 deletion. ${ }^{43}$ Studies of spontaneous $\mathrm{LOH}$ of the $H L A-A$ gene in normal human blood cells or cell lines ${ }^{44,45}$ and of spontaneous $\mathrm{LOH}$ of the adenine phosphoribosyltransferase gene in normal mice fibroblasts ${ }^{46}$ showed mitotic recombination as the primary mechanism of the second hit, although nondisjunction also contributed to spontaneous LOH.

Using SNPs, we showed that evolution from heterozygous to homozygous (diploid) KIT-activating mutation is mediated by the mechanism of nondisjunction in three GIST patients. KIT is located at $4 \mathrm{q}^{12}$, immediately adjacent to the centromere, hence spontaneous or random mitotic recombination involving KIT would be an extremely infrequent event. This may be one of the major factors leading to our observation that nondisjunction, rather than mitotic recombination, proved to be the mechanism leading to homozygous KIT mutation in all three GISTs. A common example of chromosomal nondisjunction during meiosis occurs in human embryogenesis, leading to an aneuploid embryo and subsequent fetal loss. ${ }^{47}$ The precise molecular events in mitotic nondisjunction leading to uniparental isodisomy are largely unknown, but tetraploid cells $^{48}$ or trisomy cells ${ }^{49}$ could exist initially, followed by a concomitant or subsequent loss of the normal chromosome. We propose the following molecular events: an underlying cellular defect results in a clonal occurrence of mitotic nondisjunction-nonsegregation and co-migration of the sister chromatids harboring KIT mutations during mitosis. This led to incomplete cytokinesis, which subsequently gave rise to aneuploid progenies of monosomy and trisomy. The trisomy, which retains both nonsegregated sister chromatids and a wild-type chromosome, results in uniparental disomy by concomitant or subsequent loss of the wild-type chromosome. The clones exhibiting duplicated chromosome 4, which harbored the KIT-activating mutation, prevailed and outgrew the heterozygous and other aneuploid clones.

Acquired homozygous KIT mutation likely provided significant selection advantage, resulting in total dominance of these clones within a short period of 6-13 months. Our findings suggest the presence of an underlying genetic defect in the tumor cells, ie, chromatin remodeling or genomic instability, rather than the occurrence of spontaneous random events. Further study on the underlying defect or the predisposing factor(s) that result in mitotic nondisjunction may provide additional insights into tumorigenesis and lead to the discovery of new targets for cancer therapy.

\section{Acknowledgements}

This study is supported partly by an Institutional Research Grant from The University of Texas MD Anderson Cancer Center (Lei L Chen 2004-2006);
National Cancer Institute Grants CA16672 (supporting the DNA-sequencing core facility, nucleic acid core facility and tissue procurement and banking facility), U01-CA70172-01 and N0-CM-17003 to The University of Texas MD Anderson Cancer Center and by the Goodwin Foundation. We thank all patients who participated in the laboratory protocol studies; Dr Waun Ki Hong of The University of Texas MD Anderson Cancer Center for departmental support; The ARUP Institute for Clinical and Experimental Pathology, University of Utah; Lynda J Corley for laboratory assistance and Latricia A Gratten and Dorothy Crump for article preparation.

\section{Disclosure/Conflict of Interest}

The authors have no conflicts of interest to declare.

\section{References}

1 Hirota S, Isozaki K, Moriyama Y, et al. Gain-of-function mutations of c-kit in human gastrointestinal stromal tumors. Science 1998;279:577-580.

2 Koh JS, Trent JC, Chen LL, et al. Gastrointestinal stromal tumors: overview of pathologic features, molecular biology, and therapy with imatinib mesylate. Histol Histopathol 2004;19:565-574.

3 Heinrich MC, Corless CL, Demetri GD, et al. Kinase mutations and imatinib response in patients with metastatic gastrointestinal stromal tumor. J Clin Oncol 2003;21:4342-4349.

4 Heinrich MC, Corless CL, Deunsing A, et al. PDG FRA activating mutations in gastrointestinal stromal tumors. Science 2003;299:708-710.

5 Hirota S, Ohashi A, Nishida T, et al. Gain-of-function mutations of platelet-derived growth factor receptor $\alpha$ gene in gastrointestinal stromal tumors. Gastroentero logy 2003;125:660-667.

6 Miettinen M, Lasota J. Gastrointestinal stromal tumors-review on morphology, molecular pathology, prognosis, and differential diagnosis. Arch Pathol Lab Med 2006;130:1466-1478.

7 Rubin BP. Gastrointestinal stromal tumours: an update. Histopathology 2006;48:83-96.

8 Holden JA, Willmore-Payne C, Coppola D, et al. High resolution melting amplicon analysis as a method to detect c-Kit and platelet derived growth factor receptor alpha activating mutations in gastrointestinal stromal tumors. Am J Clin Pathol 2007;128:230-238.

9 Fabbro D, Ruetz S, Buchdunger E, et al. Protein kinases as targets for anticancer agents: from inhibitors to useful drugs. Pharmacol Ther 2002;93:79-98.

10 van Oosterom AT, Judson I, Verweij J, et al. Safety and efficacy of imatinib (STI571) in metastatic gastrointestinal stromal tumours: a phase I study. Lancet 2001;358:1421-1423.

11 Demetri GD, von Mehren M, Blanke CD, et al. Efficacy and safety of imatinib mesylate in advanced gastrointestinal stromal tumors. N Engl J Med 2002; 347:472-480.

12 Chen LL, Trent JC, Wu EF, et al. A missense mutation in KIT kinase domain 1 correlates with imatinib 
resistance in gastrointestinal stromal tumors. Cancer Res 2004;64:5913-5919.

13 Chen LL, Sabripour M, Andtbacka RHI, et al. Imatinib resistance in gastrointestinal stromal tumors. Curr Oncol Rep 2005;7:293-299.

14 Tamborini E, Bonadiman L, Greco A, et al. A new mutation in the KIT ATP pocket causes acquired resistance to imatinib in a gastrointestinal stromal tumor patient. Gastroenterology 2004;127:294-299.

15 Wakai T, Kanda T, Hirota S, et al. Late resistance to imatinib therapy in a metastatic gastrointestinal stromal tumour is associated with a second KIT mutation. Br J Cancer 2004;90:2059-2061.

16 Antonescu CR, Sommer G, Sarran L, et al. Association of KIT exon 9 mutations with nongastric primary site and aggressive behavior: KIT mutation analysis and clinical correlates of 120 gastrointestinal stromal tumors. Clin Cancer Res 2003;9:3329-3337.

17 Knudson AG. Antioncogenes and human cancer. Proc Natl Acad Sci USA 1993;90:10914-10921.

18 Weinberg RA. Tumor suppressor genes. Science 1991; 254:1138-1146.

19 Levine RL, Wadleigh M, Cools J, et al. Activating mutation in the tyrosine kinase JAK2 in polycythemia vera, essential thrombocythemia, and myeloid metaplasia with myelofibrosis. Cancer Cell 2005;7:387-397.

20 Raghavan M, Lillington DM, Skoulakis S, et al. Genome-wide single nucleotide polymorphism analysis reveals frequent partial uniparental disomy due to somatic recombination in acute myeloid leukemias. Cancer Res 2005;65:375-378.

21 Murthy SK, DiFrancesco LM, Ogilvie RT, et al. Loss of heterozygosity associated with uniparental disomy in breast carcinoma. Mod Pathol 2002;15:1241-1250.

22 Tung WS, Shelvin DW, Kaleem Z, et al. Allelotype of follicular thyroid carcinomas reveals genetic instability consistent with frequent nondisjunctional chromosomal loss. Genes Chromosomes Cancer 1997;19: $43-51$.

23 Steigen SE, Eide TJ, Wasag B, et al. Mutations in gastrointestinal stromal tumors-a population-based study from Northern Norway. APMIS 2007;115: 289-298.

24 Lasota J, vel Dobosz AJ, Wasag B, et al. Presence of homozygous KIT exon 11 mutations is strongly associated with malignant clinical behavior in gastrointestinal stromal tumors. Lab Invest 2007;87: 1029-1041.

25 Corless CL, Fletcher JA, Heinrich MC. Biology of gastrointestinal stromal tumors. J Clin Oncol 2004;22:3813-3825.

26 Lin W, Yang HH, Lee MP. Allelic variation in gene expression identified through computational analysis of the dbEST database. Genomics 2005;86: 518-527.

27 Willmore C, Holden JA, Zhou L, et al. Detection of c-kit-activating mutations in gastrointestinal stromal tumors by high-resolution amplicon melting analysis. Am J Clin Pathol 2004;122:206-216.

28 Lasota J, Dansonka-Mieszkowska A, Stachura T, et al. Gastrointestinal stromal tumors with internal tandem duplications in $3^{\prime}$ end of KIT juxtamembrane domain occur predominantly in stomach and generally seem to have a favorable course. Mod Pathol 2003;16: 1257-1264.

29 Corbacioglu S, Kilic M, Westhoff MA, et al. Newly identified c-KIT receptor tyrosine kinase ITD in child- hood AML induces ligand-independent growth and is responsive to a synergistic effect of imatinib and rapamycin. Blood 2006;108:3504-3513.

30 London CA, Galli SJ, Yuuki T, et al. Spontaneous canine mast cell tumors express tandem duplications in the proto-oncogene c-kit. Exp Hematol 1999;27: 689-697.

31 Nakao M, Yokota S, Iwai $\mathrm{T}$, et al. Internal tandem duplication of the flt3 gene found in acute myeloid leukemia. Leukemia 1996;10: 1911-1918.

32 Yokota S, Kiyoi H, Nakao M, et al. Internal tandem duplication of the FLT3 gene is preferentially seen in acute myeloid leukemia and myelodysplastic syndrome among various hematological malignancies. A study on a large series of patients and cell lines. Leukemia 1997;11:1605-1609.

33 Kikuchi H, Yamashita K, Kawabata $\mathrm{T}$, et al. Immunohistochemical and genetic features of gastric and metastatic liver gastrointestinal stromal tumors: sequential analyses. Cancer Sci 2006;97: 127-132.

34 Simioni P, Scudeller A, Radossi P, et al. 'Pseudo homozygous' activated protein $\mathrm{C}$ resistance due to double heterozygous factor $\mathrm{V}$ defects (factor $\mathrm{V}$ Leiden mutation and type I quantitative factor $\mathrm{V}$ defect) associated with thrombosis: report of two cases belonging to two unrelated kindreds. Thromb Haemost 1996;75:422-426.

35 Brugge JM, Simioni P, Bernardi F, et al. Expression of the normal factor $\mathrm{V}$ allele modulates the APC resistance phenotype in heterozygous carriers of the factor V Leiden mutation. J Thromb Haemost 2005;3: 2695-2702.

36 Kim HJ, Lim S, Park K, et al. Multiple gastrointestinal stromal tumors with a germline $c$-kit mutation. Pathol Int 2005;55:655-659.

37 O'Riain C, Corless CL, Heinrich MC, et al. Gastrointestinal stromal tumors: insights from a new familial GIST kindred with unusual genetic and pathologic features. Am J Surg Pathol 2005;29: 1680-1683.

38 Corless CL, McGreevey L, Haley A, et al. KIT mutations are common in incidental gastrointestinal stromal tumors one centimeter or less in size. Am J Pathol 2002;160:1567-1572.

39 Mol CD, Dougan DR, Schneider TR, et al. Structural basis for the autoinhibition and STI-571 inhibition of c-Kit tyrosine kinase. J Biol Chem 2004;279: 31655-31663.

40 Chen LL, Prieto VG, Sabripour M, et al. Loss of heterozygosity with acquisition of homozygous KITactivating mutation promotes gastrointestinal stromal tumor progression [Abstract]. Proc Am Soc Clin Oncol 2006;23:9530.

41 Haigis KM, Dove WF. A Robertsonian translocation suppresses a somatic recombination pathway to loss of heterozygosity. Nat Genet 2003;33:33-39.

42 Tischfield JA, Shao C. Somatic recombination redux. Nat Genet 2003;33:5-6.

43 Zhang J, Lindroos A, Ollila S, et al. Gene conversion is a frequent mechanism of inactivation of the wild-type allele in cancers from MLH1/MSH2 deletion carriers. Cancer Res 2006;66:659-664.

44 de Nooij-van Dalen AG, van Buuren-van Seggelen VH, Lohman PH, et al. Chromosome loss with concomitant duplication and recombination both contribute most to 
loss of heterozygosity in vitro. Genes Chromosomes Cancer 1998;21:30-38.

45 Morley AA, Grist SA, Turner DR, et al. Molecular nature of in vivo mutations in human cells at the autosomal HLA-A locus. Cancer Res 1990;50: 4584-4587.

46 Shao C, Deng L, Henegariu O, et al. Mitotic recombination produces the majority of recessive fibroblast variants in heterozygous mice. Proc Natl Acad Sci USA 1999;96:9230-9235.
47 Lamb NE, Hassold TJ. Nondisjunction-a view from ringside. N Engl J Med 2004;351: 1931-1934.

48 Shi Q, King RW. Chromosome nondisjunction yields tetraploid rather than aneuploid cells in human cell lines. Nature 2005;437:1038-1042.

49 Minissi S, Degrassi F, Tanzarella C, et al. Direct and indirect non-disjunction in the origin of trisomy in cultured human lymphocytes. Mutagenesis 1999;14: $557-562$. 